\title{
The effects of recombinant human parathyroid hormone, rhPTH(1-84), on bone mass in undernourished rats
}

\author{
Y Rhee, R Namgung ${ }^{1}$, D-H Park ${ }^{2}$, H C Lee, G B Huh and S-K Lim \\ Department of Internal Medicine, College of Medicine, Yonsei University, 134 Shinchon-Dong, Seodaemoon-Gu, Seoul 120-752, Korea \\ ${ }^{1}$ Department of Pediatrics, College of Medicine, Yonsei University, Seoul, Korea \\ ${ }^{2}$ Mogam Biotechnology Research Institute, Kyungi, Korea \\ (Requests for offprints should be addressed to S-K Lim; Email: Isk@yumc.yonsei.ac.kr)
}

\begin{abstract}
Energy intake restriction reduces bone formation both in protein-energy malnourished children and in undernourished rats, and such conditions might cause partial or irreversible bone loss. Because the use of anti-resorptive agents in this situation is seemingly limited, we examined the effect of the anabolic agent, recombinant human parathyroid hormone ( $\operatorname{rhPTH}(1-84))$, on bones in undernourished conditions. First, the osteopenic changes of rat bones with $40 \%$ restricted diet for 4 weeks were confirmed. Subsequently, another set of the rats were randomized into four groups and studied for 8 weeks: the freely fed group (control group); the restricted diet, then freely fed group (restriction-ad libitum group); the restricted diet-vehicle-treated group (restriction-vehicle group); and the restricted diet-PTH-treated group (restriction-PTH group). In the restriction-vehicle group, total femoral bone mineral density (BMD) was lower and femoral length was shorter than the control group
\end{abstract}

by $15 \cdot 4 \%$ and $8 \cdot 1 \%$ respectively $(P<0 \cdot 05)$. In the restriction-ad libitum group, these parameters recovered fully to those of the control group. In the case of intermittent $\mathrm{PTH}$ treatment in the persistent undernourished state, the BMD of total femur caught up with those of the control or the restriction-ad libitum group. However, the femoral length remained shorter than those of the other groups. Serum osteocalcin was significantly reduced in continuously undernourished rats, whereas it was elevated in the restriction-PTH group. In conclusion, BMD of total femur was low in undernourished rats. However, it increased after re-feeding ad libitum or intermittent PTH treatment. We suggest that rhPTH(1-84) may be a possible therapeutic agent for ongoing bone loss, especially in patients in a chronically undernourished condition.

Journal of Endocrinology (2002) 174, 419-425

\section{Introduction}

Nowadays, simple undernourishment is not as common as it was in the past, though conditions inducing cachexia such as anorexia nervosa and cancer are hard to overlook. Moreover, the restriction of energy intake is known to reduce bone formation both in malnourished children and undernourished rats (Ndiaye et al. 1995a,b). The prevalence of low bone mass in anorexia nervosa is about $50 \%$, and occurs relatively early. In untreated anorexia nervosa patients, approximately $4-10 \%$ of bone loss occurs each year (Maugars et al. 1996). Furthermore, the affected age of anorexia nervosa patients overlaps with the period of peak bone formation. The risk of lifetime fracture in anorexia nervosa patients is about 2.9 times the norm (Lucas et al. 1999).

The characteristic bone biochemical marker profiles in protein-energy malnourished children and in restrictedfed rats show a reduced level of bone formation marker
(Ndiaye et al. 1995a,b). Osteocalcin levels are also reduced in anorexia nervosa patients, and an elevation in the bone resorption markers, deoxypyridinoline (Dpd) or N-telopeptide (NTX) has been observed in anorexia nervosa (Grinspoon et al. 1996). Even after the administration of estrogen or the recovery of near normal body weight and menstruation, bone loss was only partially reversed or sometimes bone density is not recovered at all (Rigotti et al. 1991, Klibanski et al. 1995, Baker et al. 2000). Anti-resorptive agents such as estrogen and bisphosphonate, etc. have limited effects on bone changes related to anorexia nervosa (Klibanski et al. 1995). The effects of anabolic agents such as parathyroid hormone $(\mathrm{PTH})$ have not been studied in anorexia nervosa. The aim in the present study was to determine whether the intermittent administration of $\mathrm{PTH}$ would be effective on bones in persistently undernourished state.

When PTH is administered as a constant infusion to normal animals or those in a disease state such as 
hyperparathyroidism, where its level is constantly elevated, bone is lost, especially at cortical sites (Dobnig \& Turner 1997). In contrast, when the hormone is treated in an intermittent fashion, it has been reported to strongly increase lumbar bone mineral density (BMD) both in the normal and the ovariectomized rats (Gunness-Hey \& Hock 1984, Hock et al. 1988, Hori et al. 1988, Liu et al. 1991, Kimmel et al. 1993, Li et al. 1995).

Therefore, it is hypothesized that recombinant human parathyroid hormone, $\operatorname{rhPTH}(1-84)$, a strong anabolic agent, would restore bone loss related to malnourishment. To test the effects of $\mathrm{rhPTH}(1-84)$ on the bone, an undernourished osteopenic rat model was established and the resulting rats were treated with $\operatorname{rhPTH}(1-84)$ intermittently.

\section{Materials and Methods}

\section{Animals and food intake}

Ten-week-old virgin female Sprague-Dawley rats were purchased from the Department of Laboratory Animal Medicine at Yonsei Medical Research Center (Seoul, Korea). They were housed in a room maintained at $22 \pm 2{ }^{\circ} \mathrm{C}$ and on $12 \mathrm{~h}$ light : $12 \mathrm{~h}$ darkness cycles. The animals were fed in two different ways: a freely fed group and a group that was given $40 \%$ of the total amount of the food intake by the freely fed group. Purina laboratory rodent chow (Hagribrand Purina Korea Co., Kunsan, Korea) that contained $1 \cdot 17 \%$ calcium, $0 \cdot 77 \%$ phosphorous, and 2.6 IU vitamin D per gram was used. The total daily food intake of the control group was $16 \cdot 8 \pm 0 \cdot 3 \mathrm{~g}$ during the first 4 weeks and $17 \cdot 1 \pm 0.6 \mathrm{~g}$ during the last 4 weeks. Water was provided and animals allowed to drink ad libitum. All animals were treated in accordance with the guidelines and regulations for the use and care of animals of Yonsei University, Seoul, Korea.

\section{Experimental protocols}

The animals were weight-matched and divided into six groups when they were 11 weeks old. There were five to seven rats per group, and housed two rats per cage. The six groups were as follows: the 4-week control group, freely fed for 4 weeks and then sacrificed; the restriction group, a $40 \%$ restricted diet for 4 weeks prior to sacrifice; the 8 -week control group where rats were freely fed for 8 weeks then sacrificed; the restriction-ad libitum group, fed on a $40 \%$ restricted diet for the first 4 weeks and then freely fed for another 4 weeks, then sacrificed; the restriction-vehicle group, a $40 \%$ restricted diet for the whole 8 weeks and treated with a vehicle during the last 4 weeks, then sacrificed; and the restriction-PTH group, a $40 \%$ restricted diet for 8 weeks and treated with rhPTH(1-84) during the last 4 weeks, then sacrificed.
Table 1 Experimental protocols. Words shown on the lines designate the time period where vehicle or PTH was given to the assigned groups. PTH, rhPTH(1-84) (150 $\mu \mathrm{g} / \mathrm{kg}$ per day s.c.). Ad libitum diet is expressed with a bold line and $40 \%$ restricted diet with a dotted line

\begin{tabular}{lll} 
Time (weeks) \\
\hline $0 \quad 4$ & 8 \\
\hline
\end{tabular}

\section{Groups}

4 weeks

Ad libitum

Restriction

8 weeks

Ad libitum

Restriction-ad libitum

Restriction-vehicle

Restriction-PTH

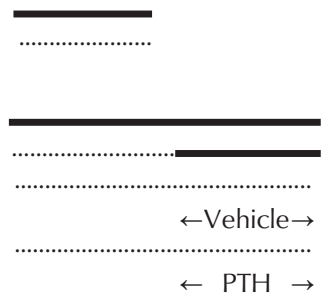

rhPTH(1-84) was a gift from the Mogam biotechnology research institute (Kyungi, Korea), dissolved in 0.9\% normal saline. The restriction-PTH group was treated daily for 5 days per week with intermittent subcutaneous injections of $150 \mu \mathrm{g} / \mathrm{kg} \operatorname{rhPTH}(1-84)$ per day. The restriction-vehicle group was injected subcutaneously with an equivalent volume of $0.9 \%$ normal saline at the same frequency. The detailed protocol used is shown in Table 1. At the end of the fourth and eighth weeks of experimental period, the rats were sacrificed by exsanguinations from the abdominal aorta under ketamine/xylazine anesthesia. Urine was collected via metabolic cages over $24 \mathrm{~h}$ at timed intervals. Rat blood samples were taken at timed intervals. Both the collected urine and serum were stored at $-20{ }^{\circ} \mathrm{C}$.

\section{Measurement of bone length and density}

The right femur lengths were measured using a Mitutoyo digital caliper (Cole Parmer, Vernon Hills, IL, USA). The femoral length was measured from the greater trochanter to intercondylar fossa. The BMD of the excised femurs was measured by dual energy X-ray absorptiometry (QDR-4500A, Hologic, Waltham, MA, USA). Triplicate determinations of the five different femurs with repositioning showed a coefficient of variation of $0.59 \%$.

\section{Osteocalcin and N-telopeptide assays}

The osteocalcin concentration of the rat serum was measured using a rat osteocalcin IRMA assay kit (Immutopics Inc., San Clemente, CA, USA). The urinary cross-linked N-telopeptide of type 1 collagen (NTX) was measured by ELISA (Osteomark, Ostex International, Seattle, WA, USA). Intra-assay coefficients of variation 
$(\mathrm{CV})$ for rat osteocalcin were $2.0 \%$ at $1.5 \mathrm{ng} / \mathrm{ml}$ and $2.3 \%$ at $4.3 \mathrm{ng} / \mathrm{ml}$, and the inter-assay $\mathrm{CV}$ was $4.0 \%$ at $4.0 \mathrm{ng} / \mathrm{ml}$. The intra-assay CVs for NTX were $18 \%$ at $26 \mathrm{nM}$ of bone collagen equivalents (BCE), $10 \%$ at $439 \mathrm{nM}$ of $\mathrm{BCE}$ and $8 \%$ at $690 \mathrm{nM}$ of BCE, and the inter-assay CV for NTX was $6 \%$ at $80 \mathrm{nM}$ of BCE. Urine creatinine was measured by Jaffe's reaction using a Hitachi 747 autoanalyzer (Boehringer Mannheim, Mannheim, Germany). The intra-assay CV was less than 3.0\% for the urine tests. The groups that were experimented on after 4 weeks were excluded in the measurement of the biochemical markers.

\section{Cortisol and estradiol assays}

The cortisol and estradiol concentration of rat serum were measured using a rat cortisol RIA assay kit (Beckman Coulter Inc., Marseille, France) and estradiol RIA assay kit (Beckman Coulter Inc.). Intra- and inter-assay CVs for rat cortisol were $2 \cdot 2 \%$ at $407 \mathrm{nmol} / 1$ and $4 \cdot 6 \%$ at $416.5 \mathrm{nmol} / 1$ respectively, and intra- and inter-assay CVs for estradiol were $7 \cdot 2 \%$ at $64 \mathrm{pg} / \mathrm{ml}$ and $7 \cdot 9 \%$ at $57 \mathrm{pg} / \mathrm{ml}$. These parameters were only measured in rats killed after 4 weeks.

\section{Statistical analysis}

SAS software (6.12 for Windows; SAS, Cary, NC, USA) was used. The data were expressed as the means \pm S.D. The differences in the BMD and femoral length between the groups were compared using ANOVA with a Bonferroni's multiple group comparison procedure. The data on body weight, serum osteocalcin and NTX at the timed intervals were analyzed by repeated measures of the ANOVA method. The difference between the osteocalcin and NTX concentration were analyzed by the Kruskal-Wallis test and compared with the results of the Mann-Whitney $U$ test. A $P$ value of less than 0.05 was accepted as statistically significant.

\section{Results}

\section{Changes of body weight}

Even after 4 weeks of a restricted diet, body weight reduction was obvious. After 8 weeks of dietary control, the animals fed a continuously restricted diet showed 51\% lower body weight than the freely fed control group $(P<0 \cdot 001$, Fig. 1$)$. The initial reduction in body weight after the restricted diet was recovered to the levels of those of the control group after 4 weeks of free feeding.

\section{Rat cortisol and estradiol concentrations}

Rat cortisol concentrations were significantly reduced after 4 weeks of restricted diet $(P<0 \cdot 05)$, but there were no

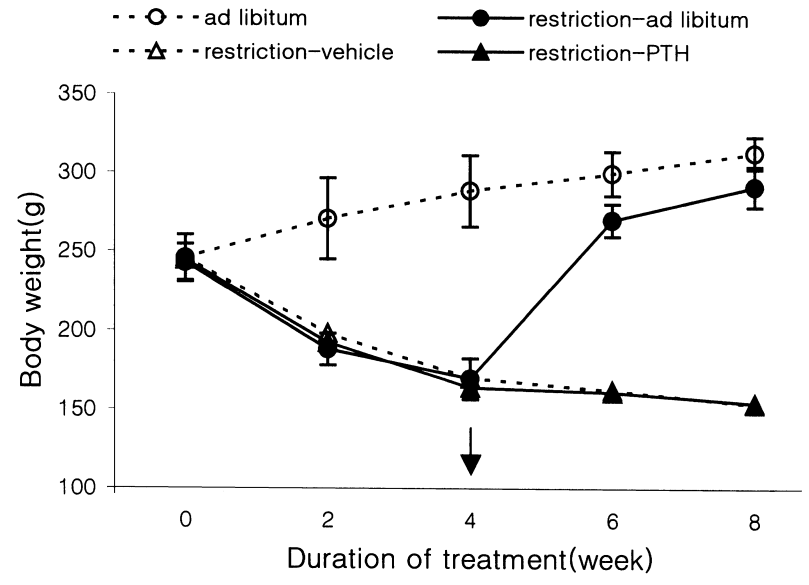

Figure 1 Effects of dietary control and PTH treatment on body weight. The rats were 11 weeks old when the study started. Values are presented as means \pm S.D. Two groups with restricted diet were injected with vehicle and $\mathrm{rhPTH}(1-84)$ respectively from the 4 th week (arrow) to the 8 th week. $P<0 \cdot 001$, by repeated measures ANOVA.

significant differences in the estradiol levels of freely fed and undernourished rats (data not shown).

\section{Femoral length}

The femoral lengths after 8 weeks in the groups that had been provided with a $40 \%$ restricted diet were significantly shorter than in the control group, and also shorter than those of the restriction-ad libitum group (Fig. 2 and Table 2). Four weeks of restricted diet was found to result in a reduced femoral length. The femoral length reduction was reversed after feeding ad libitum for a period of 4 weeks to a similar length as that of the control group. However, the administration of $\operatorname{rhPTH}(1-84)$ had no effect on the femoral length.

\section{BMD of right femur}

BMD decreased after 4 weeks of a restricted diet, but this was without statistical significance (data not shown), however, after 8 weeks the BMD was significantly lower than in the freely fed group $(P<0 \cdot 05$, Fig. 3 and Table 2$)$. The BMD was significantly increased to the level of the control group in the restriction-PTH group, and also in the restriction-ad libitum group $(P<0 \cdot 05$, Fig. 3 and Table $2)$. The body weight was positively correlated with the BMD $(r=0 \cdot 678 ; P<0 \cdot 0001)$.

\section{Serum osteocalcin}

Serum osteocalcin concentrations tend to decrease with increasing age in all groups except the restriction-PTH group (Table 3). In the restriction-PTH group, the serum 


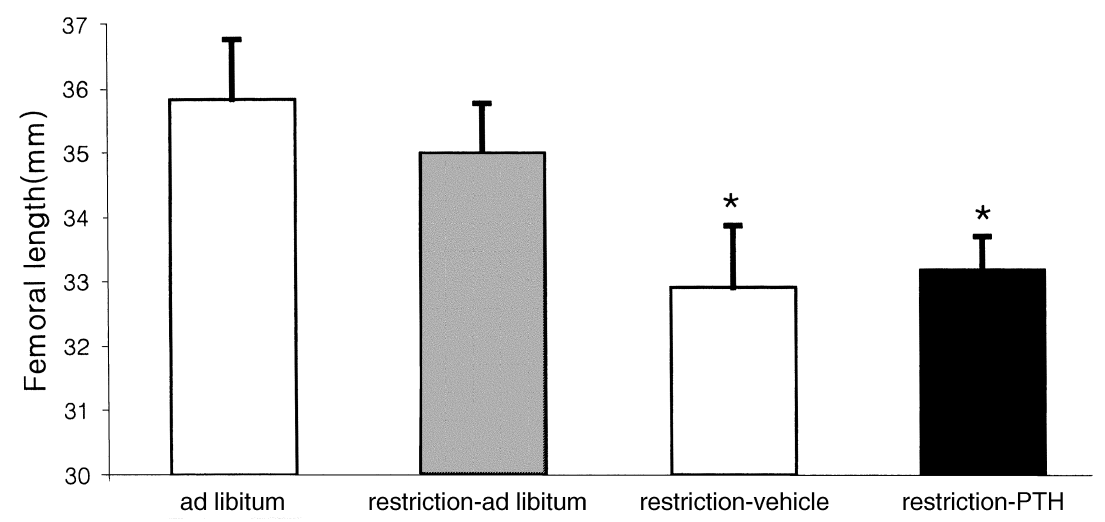

Figure 2 Effects of dietary control and PTH treatment on right femoral length at the 8th week. Each bar represents the mean \pm S.D. ${ }^{*} P<0 \cdot 01$ vs ad libitum group or restriction-ad libitum group.

osteocalcin concentration at the eighth week was increased significantly compared with those of the restrictionvehicle group $(P<0 \cdot 05$, Table 3$)$. In the restriction-ad libitum group, the serum osteocalcin concentration showed slight increasing tendency without statistical significance.

\section{NTX levels in 24-h urine}

No differences in the NTX levels in the 24-h urine were found among the groups (Table 4).

\section{Discussion}

In this study, it was found that a restricted diet in growing rats significantly reduced the body weight, linear growth and bone formation, and led to low BMD. PTH treatment significantly improved the BMD and markedly increased the serum osteocalcin levels even in the group that was maintained on a restricted diet. In the group with nutritional recovery, both the BMD and the body weight recovered significantly.
Undernourishment affects the bone metabolism detrimentally. Data on energy-protein malnourished children or undernourished rats has shown that the bone formation marker osteocalcin is present at reduced levels (Ndiaye et al. 1995a,b). The calcium content of rat bones that have suffered food restriction of $60 \%$ was significantly decreased (Kalu et al. 1988). The analysis of histomorphometric data has also shown decreased mineral apposition rate in the bones of rats fed on energy-restricted diets (Ndiaye et al. 1995a). More astonishingly, anorexia nervosa leads to partially reversible or sometimes irreversible bone loss, even after recovering the body weight to a normal level and the resumption of menstruation, or even after hormonal replacement therapy (Rigotti et al. 1991, Klibanski et al. 1995, Baker et al. 2000).

The exact mechanism of bone loss in malnourishment is not clear. However, several plausible factors have been proposed. The synthesis of insulin-like growth factor-I (IGF-I) in the bone as well as in the liver has been found to be significantly reduced in patients with anorexia nervosa (Canalis et al. 1989, Counts et al. 1992, Soyka et al. 1999). Especially in anorexia nervosa, estrogen deficiency

Table 2 Effects of dietary control and PTH treatment on femoral length and density. Values are means \pm S.D.

\begin{tabular}{|c|c|c|c|c|}
\hline & \multicolumn{4}{|l|}{8 weeks } \\
\hline \multicolumn{5}{|l|}{ Parameters } \\
\hline$n$ & 6 & 6 & 6 & 6 \\
\hline Femoral length (mm) & $35.83 \pm 0.93$ & $34.99 \pm 0 \cdot 77$ & $32 \cdot 90 \pm 0 \cdot 96^{a}$ & $33 \cdot 17 \pm 0 \cdot 52^{a}$ \\
\hline $\mathrm{BMC}(\mathrm{g})$ & $0.479 \pm 0.004$ & $0 \cdot 440 \pm 0 \cdot 004$ & $0 \cdot 347 \pm 0 \cdot 004$ & $0 \cdot 397 \pm 0 \cdot 002$ \\
\hline Area $\left(\mathrm{cm}^{2}\right)$ & $1 \cdot 821 \pm 0 \cdot 120$ & $1.737 \pm 0.006$ & $1 \cdot 555 \pm 0.009^{b}$ & $1 \cdot 612 \pm 0.038^{\mathrm{c}}$ \\
\hline
\end{tabular}

${ }^{\mathrm{a}} \mathrm{P}<0.001 \mathrm{vs}$ ad libitum and restricted-ad libitum diet groups; ${ }^{\mathrm{b}} \mathrm{P}<0.05 \mathrm{vs}$ ad libitum and restricted-ad libitum diet groups; ${ }^{\mathrm{c}} P<0 \cdot 05$ vs ad libitum; ${ }^{\mathrm{d}} P<0 \cdot 05$ vs ad libitum, restricted-ad libitum diet and restricted diet-PTH-treated groups.

$\mathrm{BMC}$, bone mineral content. 


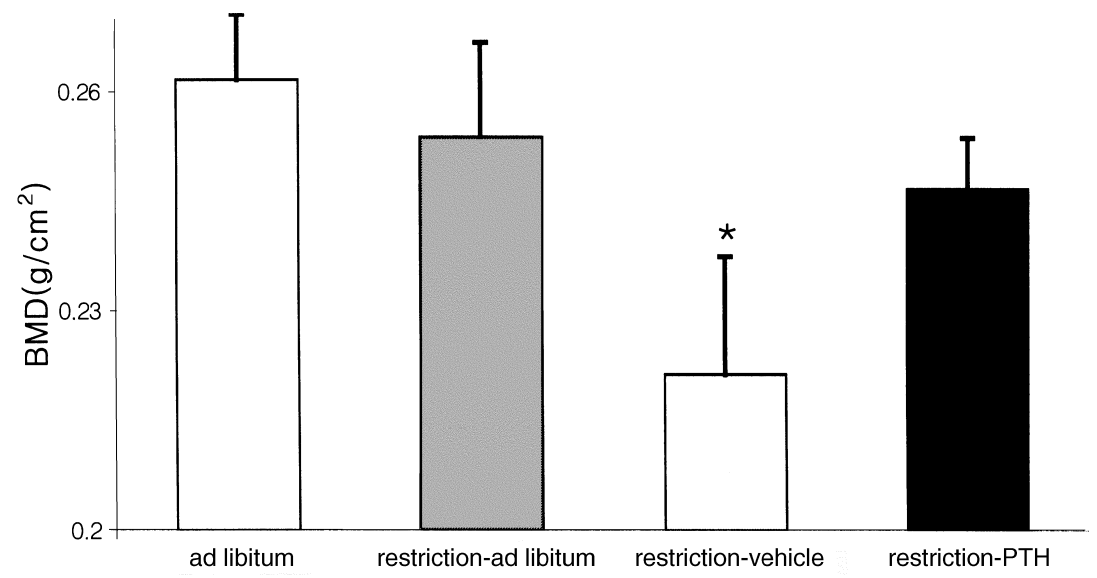

Figure 3 Effects of dietary control and PTH treatment on BMD of right total femur at the 8th week. Each bar represents mean \pm S.D. ${ }^{*} P<0.01 \mathrm{vs}$ ad libitum group and restriction-ad libitum group and $P<0 \cdot 05$ vs restriction-PTH group.

is one of the contributing factors, because the degree of bone loss is directly correlated with the duration of amenorrhea (Biller et al. 1989). Hypercortisolism, present in about $22 \%$ of patients, can also accelerate bone loss (Lucas et al. 1999). Another worrisome problem might be an increase in the PTH concentration during food restriction. However, Kalu et al. showed that there was no significant change or difference in PTH between freely fed and restricted-diet rat groups (Kalu et al. 1988).

In undernourished children, it was shown that reduced osteocalcin levels are fully normalized during nutritional recovery (Prudhon et al. 1991). However, in conditions such as anorexia nervosa, several trials aimed at preventing bone loss in these patients have been conducted in vain. Neither daily calcium supplements for 23 months nor estrogen replacement for 1 year increased the BMD in osteopenic patients with anorexia nervosa (Rigotti et al. 1991, Klibanski et al. 1995). A 6-day trial of low dose rhIGF-I in women with anorexia nervosa increased the carboxyl-terminal propeptide of the type 1 procollagen (PICP) by up to $21.6 \%$, without any evidence of enhanced bone resorption (Lucas et al. 1999). However, the clinical application of IGF-I has several limitations, such as short plasma half-life and some side effects, including hypoglycemia and parotid swelling (Guler et al. 1989, Kovacs

Table 3 Changes of serum osteocalcin. Values are means \pm S.D. $(\mathrm{ng} / \mathrm{ml})$

\begin{tabular}{|c|c|c|c|}
\hline & Initial & Fourth week & Eighth week \\
\hline Ad libitum & $53 \cdot 0 \pm 15 \cdot 9$ & $48 \cdot 3 \pm 29 \cdot 9$ & $34 \cdot 2 \pm 3 \cdot 0$ \\
\hline Restriction-ad libitum & $49 \cdot 5 \pm 13 \cdot 9$ & $26 \cdot 0 \pm 15 \cdot 1$ & $36 \cdot 1 \pm 11 \cdot 9$ \\
\hline Restriction-vehicle & $55 \cdot 1 \pm 20 \cdot 0$ & $39 \cdot 6 \pm 12 \cdot 7$ & $30 \cdot 6 \pm 4 \cdot 2$ \\
\hline Restriction-PTH & $50 \cdot 8 \pm 5 \cdot 3$ & $35 \cdot 7 \pm 9 \cdot 3$ & $59 \cdot 4 \pm 16 \cdot 8^{a}$ \\
\hline
\end{tabular}

${ }^{a} P<0.05$ vs restriction-vehicle group by Mann-Whitney $U$ test. et al. 1999). Therefore, another intervention trial to prevent or treat bone loss associated with this disorder is warranted.

Intermittent administration of PTH stimulates both bone formation and resorption and has a greater net impact on bone formation, resulting in an increase in bone mass (Gunness-Hey \& Hock 1984, Hock et al. 1988, Hori et al. 1988, Liu et al. 1991, Kimmel et al. 1993). The intermittent administration of PTH doubled the expression of the IGF-I mRNA in the osteoblasts and stimulated collagen synthesis (Watson et al. 1995, Urena et al. 1993). Recently, Jilka et al. reported that PTH suppressed osteoblasts apoptosis, resulting in prolonged survival of the osteoblasts (Jilka et al. 1999). Assuming that bone loss in undernourishment may have a deleterious effect on bone due to the chronic propensity for fracture, the aim of this study was to determine whether or not intermittent PTH treatment could enhance BMD in a malnourished state. The calorie intake was restricted to about $40 \%$ of the quantity normally consumed by the control rat group in this experiment. The rationales for reducing the calorie intake to $40 \%$ in this experiment were as follows. Patients with anorexia nervosa are known to consume approximately $60 \%$ of the daily intake of the controls, without any significant differences in the composition of

Table 4 Changes of 24-h urine NTX. Values are means \pm S.D. (nM $\mathrm{BCE} / \mathrm{mM}$ creatinine)

\begin{tabular}{|c|c|c|c|}
\hline & Initial & Fourth week & Eighth week \\
\hline Ad libitum & $24 \cdot 8 \pm 7 \cdot 4$ & $32 \cdot 0 \pm 14 \cdot 4$ & $21 \cdot 0 \pm 7 \cdot 0$ \\
\hline Restriction-ad libitum & $32 \cdot 8 \pm 23 \cdot 4$ & $32 \cdot 3 \pm 19 \cdot 5$ & $25 \cdot 4 \pm 7 \cdot 7$ \\
\hline Restriction-vehicle & $52 \cdot 0 \pm 44 \cdot 6$ & $86 \cdot 7 \pm 159 \cdot 9$ & $32 \cdot 3 \pm 16 \cdot 4$ \\
\hline Restriction-PTH & $21 \cdot 7 \pm 6 \cdot 8$ & $29 \cdot 8 \pm 12 \cdot 8$ & $30 \cdot 1 \pm 8 \cdot 3$ \\
\hline
\end{tabular}

Journal of Endocrinology (2002) 174, 419-425 
protein, fat and carbohydrate (Gwirtsman et al. 1989). However, in a previous animal study, only $0.9 \%$ bone loss with a concomitant $16.5 \%$ decrease in body weight was observed after restricting the calorie intake to $60 \%$ of that normally consumed by rats for 18 weeks (Banu et al. 1999).

As expected, intermittent $\mathrm{rhPTH}(1-84)$ administration increased the BMD to the level of the control group $(10 \cdot 5 \%$ vs restriction-vehicle group; $P<0 \cdot 05)$. Interestingly, nutrition enrichment alone after 4 weeks of dietary restriction also improved the BMD in rats. Our observation of an impressive increment in BMD after intermittent $\mathrm{rhPTH}(1-84)$ treatment in a persistently malnourished state is meaningful, especially with respect to potential therapies against bone loss.

While the serum osteocalcin concentration was decreased in accordance with the duration of the restricted diet and the degree of weight loss, it significantly increased after intermittent $\mathrm{rhPTH}(1-84)$ treatment. Nutritional enrichment also tends to increase the osteoblastic activity. However, no significant changes in the urinary bone resorption marker, NTX, were observed in any group. The wide distribution of the data and the high coefficient of variation in the urinary NTX concentration undoubtedly contributed to this result.

Recently, Kronenberg et al., after observing that mice were missing either the PTH-related peptide (PTHrP) or the $\mathrm{PTH} / \mathrm{PTHrP}$ receptor gene, proposed that PTHrP controls the pace of differentiation in the growth plate, and that the Indian hedgehog(Ihh)-PTHrP feedback system regulates the rate of chondrocyte differentiation, thereby balancing the growth and ossification of the long bones (Kronenberg et al. 1996). Our previous study also showed that intermittent PTH treatment promoted linear growth in ovariectomized juvenile rats ( $\mathrm{Lim}$ et al. 1999). However, in contrast to the BMD increment, linear growth did not recover to that of the control group despite rhPTH(1-84) treatment in the persistent malnourished state. This suggests that the process of linear growth is a more complex process that is regulated by many systemic and local factors under optimal nutritional conditions. However, more investigation is needed in order to determine the relationship between linear growth impairment and nutritional deprivation.

In conclusion, the intermittent administration of rhPTH(1-84) stimulated bone formation and increased the BMD in malnourished rats. It is suggested that rhPTH(1-84) may be a possible therapeutic and preventive agent for ongoing bone loss, especially for those in a chronically undernourished condition.

\section{Acknowledgements}

This work was supported by the research grant from G7 Korea Ministry of Science and Technology and from the
Brain Korea 21 project for medical sciences. We also thank Dr John Roberts for English language revision.

\section{References}

Baker D, Roberts R \& Towell T 2000 Factors predictive of bone mineral density in eating-disordered women: a longitudinal study. International Journal of Eating Disorder 27 29-35.

Banu MJ, Orhii PB, Mejia W, McCarter RJM, Mosekilde L, Thomsen JS \& Kalu DN 1999 Analysis of the effects of growth hormone, voluntary exercise, and food restriction on diaphyseal bone in female F344 rats. Bone 25 469-480.

Biller BM, Saxe V, Herzog DB, Rosenthal DI, Holzman S \& Klibanski A 1989 Mechanisms of osteoporosis in adult and adolescent women with anorexia nervosa. Journal of Clinical Endocrinology and Metabolism 68 548-54.

Canalis E, Centrella M, Burch W \& McCarthy TL 1989 Insulin-like growth factor 1 mediates selective anabolic effects of parathyroid hormone in bone cultures. Journal of Clinical Investigation $\mathbf{8 3}$ $60-65$.

Counts DR, Gwirtsman H, Carlsson LMS, Lesem M \& Cutler Jr GB 1992 The effect of anorexia nervosa and refeeding on growth hormone-binding protein, the insulin-like growth factors(IGFs), and the IGF-binding proteins. Journal of Clinical Endocrinology and Metabolism 75 762-767.

Dobnig H \& Turner RT 1997 The effects of programmed administration of human parathyroid hormone fragment (1-34) on bone histomorphometry and serum chemistry in rats. Endocrinology 138 4607-4612.

Grinspoon S, Baum H, Lee K, Anderson E, Herzog D \& Klibanski A 1996 Effects of short-term recombinant human insulin-like growth factor I administration on bone turnover in osteopenic women with anorexia nervosa. Journal of Clinical Endocrinology and Metabolism $\mathbf{8 1}$ $3864-3870$

Guler HP, Zapf J, Schmid C \& Froesch ER 1989 Insulin-like growth factors I and II in healthy man. Estimations of half-lives and production rates. Acta Endocrinologica 121 753-758.

Gunness-Hey M \& Hock JM 1984 Increased trabecular bone mass in rats treated with human synthetic parathyroid hormone. Metabolic Bone Disease and Related Research 5 177-181.

Gwirtsman HE, Kaye WH, Curtis SR \& Lyter LM 1989 Energy intake and dietary macronutrient content in women with anorexia nervosa and volunteers. Journal of the American Diabetic Association 89 $54-57$.

Hock JM, Gera I, Fonseca J \& Raisz LG 1988 Human parathyroid hormone-(1-34) increases bone mass in ovariectomized and orchidectomized rats. Endocrinology 122 2899-2904.

Hori M, Uzawa T, Morita K, Noda T, Takahashi H \& Inoue J 1988 Effect of human parathyroid hormone (PTH(1-34)) on experimental osteopenia of rats induced by ovariectomy. Bone and Mineral 3 193-199.

Jilka RL, Weinstein RS, Bellido T, Roberson P, Parfitt AM \& Manolagas SC 1999 Increased bone formation by prevention of osteoblast apoptosis with parathyroid hormone. Journal of Clinical Investigation 104 439-446.

Kalu DN, Masoro EJ, Yu BP, Hardin RR \& Hollis BW 1988 Modulation of age-related hyperparathyroidism and senile bone loss in Fischer rats by soy protein and food restriction. Endocrinology 122 $1847-1854$.

Kimmel DB, Bozzato RP, Kronis KA, Coble T, Sindrey D, Kwong P \& Recker RR 1993 The effect of recombinant human (1-84) or synthetic human (1-34) parathyroid hormone on the skeleton of adult osteopenic ovariectomized rats. Endocrinology 132 $1577-1584$

Klibanski A, Biller BMK, Shoenfeld DA, Herzog DB \& Saxe VC 1995 The effects of estrogen administration on trabecular bone loss 
in young women with anorexia nervosa. Journal of Clinical Endocrinology and Metabolism 80 898-904.

Kovacs GT, Worgall S, Schwalbach P, Steichele T, Mehls O \& Rosivall L 1999 Hypoglycemic effects of insulin-like growth factor-1 in experimental uremia: can concomitant growth hormone administration prevent this side effect? Hormone Research $\mathbf{5 1}$ 193-200.

Kronenberg HM, Karaplis AC \& Lanske B 1996 Role of parathyroid hormone-related protein in skeletal development. Annals of the New York Academy of Sciences 785 119-123.

Li M, Mosekilde L, Sogaard CH, Thomsen JS \& Wronski TJ 1995 Parathyroid hormone monotherapy and cotherapy with antiresorptive agents restore vertebral bone mass and strength in aged ovariectomized rats. Bone 16 629-635.

Lim SK, Won YJ, Park DH, Shin DH, Yook JI, Lee HC \& Huh GB 1999 Intermittent parathyroid hormone treatment can promote linear growth in the ovariectomized growing rat. Yonsei Medical Journal 40 166-172.

Liu CC, Kalu DN, Salerno E, Echon R, Hollis BW \& Ray M 1991 Preexisting bone loss associated with ovariectomy in rats is reversed by parathyroid hormone. Journal of Bone and Mineral Research $\mathbf{6}$ 1071-1080.

Lucas AR, Melton LJ III, Crowson CS \& O’Fallen WM 1999 Long-term fracture risk among women with anorexia nervosa: a population-based cohort study. Mayo Clinic Proceedings 74 972-977.

Maugars YM, Berthelot JM, Forestier R, Mammar N, Lalande S, Venisse J \& Prost AM 1996 Follow-up of bone mineral density in 27 cases of anorexia nervosa. European Journal of Endocrinology 135 591-597.
Ndiaye B, Cournot G, Pelissier M, Debray OW \& Lemonnier D $1995 a$ Rat serum osteocalcin is decreased by restriction of energy intake. Journal of Nutrition 125 1283-1290.

Ndiaye B, Lemonnier D, Sall MG, Prudhon C, Diaham B, Zeghoud F, Guillozo H, Leite N \& Wade S 1995 b Serum osteocalcin regulation in protein-energy malnourished children. Pediatric Research 37 606-610.

Prudhon C, Sall G, Ndiaye B \& Lemonnier D 1991 Nutritional regulation of serum osteocalcin: study in kwashiorkor. [in French] Comptes Rendus de l'Academie des Sciences. Serie III 313 233-238.

Rigotti NA, Neer RM, Skates SJ, Herzog DB \& Nussbaum SR 1991 The clinical course of osteoporosis in anorexia nervosa. Journal of the American Medical Association 265 1133-1138.

Soyka LA, Grinspoon S, Levitsky LL, Herzog DB \& Klibanski A 1999 The effects of anorexia nervosa on bone metabolism in female adolescents. Journal of Clinical Endocrinology and Metabolism $\mathbf{8 4}$ 4489-4496.

Urena P, Kong XF, Abou-Samra AB, Juppner H, Kronenberg HM, Potts JT Jr \& Segre GV 1993 Parathyroid hormone(PTH)/PTHrelated peptide receptor messenger ribonucleic acids are widely distributed in rat tissues. Endocrinology 133 617-623.

Watson P, Lazowski D, Han V, Fraher L, Steer B \& Hodsmon A 1995 Parathyroid hormone restores bone mass and enhances osteoblast insulin-like growth factor 1 gene expression in ovariectomized rats. Bone 16 357-365.

Received 29 April 2002

Accepted 13 May 2002 